\title{
PSYCHOLOGY AND SOCIOLOGY
}

\author{
ROBERT H. LOWIE \\ American Museum of Natural History
}

What are the relations of psychology and sociology? It is clear that the sociology of both primitive and higher civilizations yields new data for psychological interpretation. But can psychology as the older science, dealing with more fundamental phenomena, throw any light on the problems that confront the sociologist and ethnologist?

The question, even in this drastic form, is hardly absurd at the present stage of sociological and anthropological thinking. On the one hand, we find Graebner, the leader of the German historical school, resolutely turning his back on anything that savors of psychological interpretation. The sum and substance of ethnology, he tells us in his Methode der Ethnologie, is to determine the actual development of cultures; and this he forthwith outlines as the result of contact between different peoples, leading to intermixture and superposition of cultural traits. From this point of view any similarities observed in different regions must be traced to a single point of origin, for there is no criterion, no certain proof, of independent development, while cultural borrowing is not only in some cases an established historical fact, but may be considered demonstrated when a resemblance of form between the particular features compared is accompanied by a corresponding similarity of associated traits. It matters not to Graebner whether a division of society into exogamous moieties may mean one thing in Australia and quite a different thing among the Iroquois or the Tlingit. He is interested in classifying cultural results, and one moiety appears no different from another. If a bungling schoolboy by a double blunder attained the same sum as a calculating-machine, Graebner would doubtless accuse either boy or machine of copying.

Very different is the position assumed by such writers as LévyBruhl, Rivers, and Wissler. Each of them would insist that it is 
not a purely objective cultural epiphenomenon that we are dealing with in culture, but that it is precisely the subjective aspect of the problem that tempts and repays study. The opposition of the writers mentioned to current psychologizing rests on very different motives and seems to be associated with certain notions as to the hierarchy of the sciences. Precisely as many biologists now hold that vital phenomena cannot be reduced to physics and chemistry but require a distinctively biological explanation, so eminent sociologists and ethnologists now tend to believe that sociological data are sui generis and defy interpretation by individual psychology. The collective ideas encountered by the sociologist, thinks LévyBruhl, are generically different from the ideas evolved by the individual mind and obey laws other than those derived from an analysis of individual psychology. Similarly, Wissler has suggested that psychological and cultural processes belong to different levels or cycles and should be interpreted independently of each other. Rivers, to be sure, does not exclude the possibility that for an ultimate explanation of cultural data recourse may be had to psychology. Nevertheless he, too, insists that in the treatment of immediate problems we must attempt "the correlation of social phenomena with other social phenomena, and the reference of the facts of social life to social antecedents."

Anyone who has delved into the semi-popular ethnological literature of, say, the last two decades will hardly fail to sympathize in very large measure with the views just cited. The cheap plausibility about many current attempts to bring primitive or modern social thought nearer to us has been admirably exposed by LévyBruhl. Yet the trouble with many of these interpretations is not that they are psychological but rather that they are folkpsychological: they rest, not on the established results of scientific psychology or at least on points of view that have proved fruitful within that science; but rather on the sort of offhand guesswork with which in everyday life we attempt to fathom the motives and thoughts of our neighbors. And even where the ethnologist does not indulge in this form of popular psychologizing he is likely to offer as a psychological explanation what cannot by the most strenuous exertion of the will be twisted into the semblance of one. 
An example is furnished by Professor Kroeber's "psychological explanation" of kinship terminologies. Professor Kroeber has it that relatives are not classified according to social but according to psychologico-linguistic categories, which he lists accordingly. His enumeration is one of the most notable feats in the history of the subject, but in what way has it anything to do with the science of psychology? What psychological processes cause many peoples to classify collateral and lineal relatives together, or to use a distinctive set of terms for a male and for a female speaking? These are linguistic phenomena that may call for a psychological interpretation; but merely to say that psychological factors have been at work is not producing the factors (such as we know from our textbooks on psychology), is not, then, a psychological explanation at all.

Yet, when all is said and done, the spirit of skepticism that has invaded sociological and ethnological circles may be carried too far. I venture to believe that some facts may not only become more intelligible when viewed from the angle of individual psychology, but it may be advisable not to defer this mode of looking at them until an indefinitely remote future. Even where individual psychology has not yet advanced far enough to give a solution of the problem, the new data may well prove a goad for further development of that branch of the science. And again an ethnologist conversant with psychology may give a more accurate description of his observations than his less sophisticated colleague.

All this becomes clearer by concrete illustration. I will begin by offering some remarks on a subject that figures largely in discussions of religion-dreams and related phenomena. Every sociologist knows of Tylor's attempt to account for the savage belief in a hereafter through visits from or to the dead as experienctd in dreams or visions. This theory can of course be assailed on ZévyBruhl's principles; it may be said that an individual will interpret his dream only in a way more or less predetermined by the mode of thought current in the society about him. But the point at issue may also be approached by the avenue of individual psychology. Some psychologists, such as Radestock and Wundt, have not hesitated to accept Tylor's theory at its face value. They find it 
perfectly natural that the thoughts of surviving relatives should continue to busy themselves with the recently deceased, and that accordingly their dream life should be haunted by the figures of those who have just departed. Nevertheless this argument is no more than a piece of plausible folk-psychologizing. Yves Delage, on the basis of personal observations, arrived at the conclusion that ideas which preoccupy the mind in waking do not appear in dreams and that one does not dream of important events of life except when the period of pre-occupation has ceased. More particularly he found that one does not tend to dream of a recently deceased relative. ${ }^{x}$ With qualifications that seem immaterial in this context Delage's views are corroborated by Professor Mary Whiton Calkins' "Statistics of Dreams." She, too, finds a strong tendency for unimportant events of waking life to crop up in dreams, while events of real significance occur with amazing infrequency; and her independent examination of dream records confirmed Delage's special point as regards the apparitions of the recently deceased.

The particular facts of this case are of course unessential. I have not followed recent dream-study sufficiently to be able to vouch for the correctness of the views cited. But one thing is clear. The sociologist who is acquainted with Delage's and Professor Calkins' observations will avoid the pitfall of a "psychological" interpretation that might otherwise seem axiomatic; the knowledge of what at least some inquirers have advanced against that interpretation will serve as a prophylactic against accepting plausible guesswork for scientific truth.

So far, to be sure, scientific psychology carries us no farther than Lévy-Bruhl's collective ideas. We have argued the merits of a particular psychological explanation from its own point of view and found it wanting; Lévy-Bruhl's principle would preclude error by simply shutting out any explanation of this type. In order to vindicate the claims of scientific psychology in sociology we must therefore prove that it has something more than a purely corrective value. This additional function consists partly in the more accurate determination of facts. Ethnological and sociological literature

x "Essai sur la théorie du rêve," Revue scientifique, XLVIII (I89r), $40 \mathrm{f}$.

'American Journal of Psychology, V (1893), 31 I-43. 
fairly reeks with such phrases as "crowd psychology," "hypnotism," "suggestion," "influence of dream life," yet rarely are these terms more than exceedingly loose and misleading catchwords. To stick to the last-mentioned topic, in ordinary savage parlance such phenomena as "dreams" and "visions" are often thrown together under a single term. Here it is the duty of the field worker to discriminate lest his record become worthless. The difference between the religious life of two tribes may center precisely in the fact that in one of them supernatural revelations are sought through artificially induced visions while in the other they come through the natural medium of dreams. And in either case by no means all the experiences are of the same significance. We know that among the central Algonkian tribes revelations through visions may be declined under the influence of the preconceived notion that a particular kind of revelation must be secured. Here, clearly enough, a collective idea overrides the individual psychological experience, but in order fully to appreciate the significance of this very fact we must know definitely what the individual experience has been, and any analogous instances from psychology and psychiatry are of value. If, on the other hand, communication with the spirit world takes place through dreams, the question arises which dreams become significant, and here an intensive psychological analysis may become necessary. An ethnologist who knows what is going on in psychology may ask whether the dreams that are culturally important conform to certain types suggested by current psychological discussion, say, whether they present the Freudian character of a repressed wish fulfilled. It may, of course, turn out that the dreams in question are wholly predetermined by social thought; but this should be the result of the investigation, not a foregone conclusion. Thus, scientific psychology may assist in greater precision of statement as to recorded facts and prevent the lumping together of disparate phenomena; and it may further suggest lines of inquiry closed to those not conversant with what psychologists are doing.

A still more important service may be rendered by psychology in connection with the ever-vexing problems of the unity or diversity of origin of similar cultural traits. Discussion of this point has 
always loomed large in the annals of anthropology; in recent times it has become the storm center in the whirlwind movements of the Graebnerian school. For, as already stated, Graebner denies that there are any objective criteria of independent development. To say, for example, that the same mythological ideas may develop independently in different parts of the world seems to him worthless, subjective twaddle. The thing is conceivable, he admits, but this does not prove that it has really taken place. Now, as I have pointed out elsewhere, ${ }^{x}$ this is true but applies in equal measure to the supposedly objective proof for historical connection. Here, too, what can be demonstrated is simply the fact that two features are similar; that such similarity means unity of origin is pure inference, not a whit less subjective than the alternative hypothesis of diversity. But in weighing the evidence pro and con we cannot but attach great significance to whatever results scientific psychology may have ascertained as to general traits of the human mind.

Take, for example, the hideous ogres that infest the mythological world of widely separated races. Shall we accept the conclusion that they took shape in a single locality and thence spread over the entire globe? If so, how did such unrealistic figments of the imagination arise? And-even if we choose to ignore the psychology of origins-why should such fantastic imagery be uncritically adopted the world over? These questions are at least answered intelligibly, if not correctly, when we learn that the unrealistic figures of mythology do occur rather frequently in dream life. For even if their origin should not be traced to this source, we can at least understand why a type of imagery familiar from dreams should be accepted as part and parcel of a conceivable mythic world. This seems, indeed, to be the verdict of psychology. Wundt distinguishes a type of dreams peopled with grotesquely distorted shapes: there are faces with enormous probosces, projecting tongues, and gnashing teeth, while the head may rest dwarffashion on a stunted body. On apparitions of this type, Wundt believes, have been patterned the Gorgons and satyrs and pygmies of mythology. ${ }^{2}$ Other students support the general psychological

I "On the Principle of Convergence in Ethnology," Journal of American Folklore, I9I 2, pp. 24-42.

${ }^{2}$ Völkerpsychologie, II, Part 2, pp. II4-I8. 
fact. Maury often saw in dreams a sort of green-winged bat with a red head and a grimace on its face. Mourly Vold reduces all these phenomena to a physiologico-psychological basis: in sleep tactile and motor sensations give rise to visual hallucinations, embracing those of the type now under consideration. ${ }^{x}$

However cautious we may be about accepting Wundt's interpretation as a final one, it is clear that the case for the theory of independent development becomes very much stronger when we find that the strange ogre figuring in myths can and does recur in individual dream life over and over again and may be referred to rather definite physiological conditions. As against Graebner we have thus scored a point. But the indefatigable disciple of LévyBruhl who is dogging our footsteps may object that when an individual dreams of, say, a Gorgon, it is because the Gorgon is a "collective idea" common to his social group, an idea with which his mind has been saturated since infancy and which thus naturally appears in his dreams. In other words, the phenomenon is essentially not psychological but sociological: as a modern philosopher inverted the commonplaces of materialism by inquiring why the mind has a body, so Lévy-Bruhl's follower nowhere sees products of individual minds becoming socialized but only social ideas shaping individual thought.

Here a twofold answer is possible. In the first place, to abandon a psychological explanation for the Gorgon is to abandon all explanation; the Gorgon enthroned as a collective idea may be inexpugnable but it is also incomprehensible and barren. Collective ideas are blind alleys; to make headway we require the admittedly fragile aircraft of individual psychology. Secondly, Lévy-Bruhl's theory involves as an essential part the doctrine of the diversity of human psychology. Why, then, we may reasonably ask, have diverse social groups produced similar mythological concepts? To this no answer is forthcoming from the opposite side.

Let us turn now to another field of inquiry. For a long time ethnologists have been struck by the odd mode of associating ideas found among primitive peoples. In some cases, to be sure, the

${ }^{\mathrm{I}}$ Vaschide, Le Sommeil et les rêves (Paris, rgrr), pp. 197-225. 
peculiarity may be due merely to our ignorance of an intermediate link that has dropped out. When I am told by a Hidatsa Indian that the maize he plants and the wild geese he shoots are one and the same thing, I am puzzled; but when I learn that both maize and wild geese are attributes of the same mythic character, a logical and possible (though not necessarily the historically correct) bond is supplied. So an educated Hindu might wonder at the emotional suggestions of the cross, but they would at once become intelligible from scriptural history. However, in most instances the search for the missing link seems hopeless; and, what is more important, the very principle of such a search seems subject to doubt. For it assumes that there is a rational bond, while the trend of modern research is certainly to emphasize not logical but "pre-logical" associations and to view the rationalistic as a secondary, superimposed feature.

Before going farther it will be well to cite some examples of the types of association I have in mind. Among the Crows I have been told that everything in the universe exists in fours. As a matter of fact, the predominance of Four as the mystic number is very striking, not only in this tribe, but throughout an immense region of North America. Processions must make four stops; songs must be sung in sets of four; mythic heroes accomplish miraculous deeds at the fourth trial, and so on. In Oregon, the place of Four is taken by Five, while in the Old World both are overshadowed by Three and Seven. M. Lévy-Bruhl has well expressed the essential fact in all these cases by describing the mystic numbers as categories into which reality is fitted: "Au lieu que le nombre dépende de la pluralité réelle des objets perçus ou imaginés, ce sont au contraire les objets dont la pluralité se définit en recevant sa forme d'un nombre mystique fixé d'avance."x

But it is not merely numbers that are associated with apparently fanciful ideas. To a Crow a diamond represents a navel cord; a rectangle, quadrilateral, right angle, and certain combinations of figures suggest to the Arapaho the notion of life and prosperity; and among the western Dakotas a form of lozenge symbolizes the whirlwind.

× Les Fonctions mentales dans les sociêtês inférieures, pp. $256 \mathrm{f}$. 
Finally (for our present purpose), there are strange associations with color. In addition to color associations that are selfexplanatory, such as the connection between red and blood, white and snow, green and grass, there are others of a puzzling character. In several Plains tribes black symbolizes victory and joy; the Cherokees associate white with the south, red with the east, black with the west, and blue with the north; the Dakotas symbolize both the north and the south by blue, etc.

How are we to account for such associations? The interpretations usually given are manifestly unsatisfactory. It will not do, for example, to say that geometrical designs are derived from realistic representations of objects in nature through a process of degeneration, the name of the original model having been retained for the conventionalized, geometrical form. First of all, this does not account for symbolism of an abstract character. Secondly, it has been found that often the same pattern symbolizes one thing in one tribe and another in a neighboring tribe, or even different things within the same tribe. As for numbers, it has been suggested that the mystic qualities of Four are due to the existence of four cardinal directions and winds, the idea of which is again associated with four sacred animals, colors, and what not. Lévy-Bruhl has given an admirable critique of this and other so-called psychological theories of the same nature. In the primitive mind, he argues, there is no conception of north as a spatial division, with west at the left and east at the right, to which there are subsequently added the ideas of cold winds, snow, the bear, blue, etc. Rather are all these ideas bound up in a single complex collective idea, with the mystic elements masking those which we call real, and within this complex is comprised the element Four. When the mystical "participations" are no longer felt, there are precipitated the associations that everywhere persist in some measure. Now they are associations because the inner bond that integrated them has disappeared; but originally they were of quite a different character. ${ }^{\mathrm{I}}$ Again, the mystic properties of Seven among the Malays have been derived from the fact that the Malays believe that man has seven souls. Arguing in a way that must be absolutely convincing to every unbiased

× Lévy-Bruhl, op. cit., p. 242. 
ethnological thinker, Lévy-Bruhl inverts this supposed explanation. Seven does not play the part of the mystic number because the Malay believes in seven souls, but the Malay believes in seven souls because the pre-existing numerical category predetermines his speculations as to the number of souls. ${ }^{x}$

Nevertheless this point of view cannot be a final one. It may be that the Malay conception of Seven has been an established category for untold aeons, and that the complex collective idea of Four is of corresponding antiquity in North America. Nevertheless, somehow and somewhere these complex "collective ideas" must have taken shape in an individual mind; to "explain" them psychologically, i.e., to class them with related phenomena of individual psychology, seems to be indispensable for a proper understanding of the facts.

In his Inquiries into Human Faculty and Its Development, Francis Galton has collected data at least generically related, I believe, to those under discussion. He found that imaginative persons almost invariably think of numerals in some form of visual imagery.

If the idea of six occurs to them, the word "six" does not sound in their mental ear, but the figure 6 in a written or printed form rises before their mental eye. . . . . Those who are able to visualize a numeral with a distinctness comparable to reality, and to behold it as if it were before their eyes, and not in some sort of dreamland, will define the direction in which it seems to lie, and the distance at which it appears to be. If they were looking at a ship on the horizon at the moment that the figure 6 happened to present itself to their minds, they could say whether the image lay to the left or right of the ship, and whether it was above or below the line of the horizon; they could always point to a definite spot in space, and say with more or less precision that that was the direction in which the image of the figure they were thinking of first appeared.

To a person of this type, series of numbers arrange themselves "in a definite pattern that always occupies an identical position in his field of view with respect to the direction in which he is looking." These patterns or "forms" vary individually, but are stated in all cases to date as long back as the memory extends, to come into view independently of the will, and to be nearly constant for a given individual. Moreover, there is the strongest evidence that the

${ }^{2}$ Ibid., p. 250. 
peculiarity is hereditary "after allowing and over-allowing for all conceivable influences of education and family tradition."

Galton discovered not only an association between series of numbers and definite patterns, but an additional association, in some cases, between series of numbers and colors. And what is perhaps of still greater immediate interest for the present purpose, he found that numbers are often personified and invested with a definite character. Three was described by different informants, respectively, as a treacherous sneak, a good old friend, delightful and amusing, etc. Galton himself "had absurdly enough fancied that of course the even numbers would be taken to be of the male sex, and was surprised to find that they were not." The association of color with sounds had been known prior to Galton. Galton notes cases of the association of definite colors with certain letters and with certain days of the week. One of his correspondents not only associated letters with colors, but conversely collected "scraps of various patterns of wall paper, and sent them together with the word that the colour of the several patterns suggested to him." A blue bottle-shaped design on a like background suggested "sweet," yellow leaves on a yellow-red background striated with black vertical lines meant "range."

The psychological phenomena presented by Galton seem to me, I repeat, connected with the cultural phenomena under discussion. The association between a blue bottle design and sweetness does not seem to differ generically from the Dakota's association of a lozenge with the whirlwind. If an English-woman thinks of Tuesday in association with a gray sky color, while Friday suggests a dull yellow smudge, why should not the Indian associate the north with blue and the south with white? And if numbers are endowed with individual personalities by Europeans, ${ }^{x}$ what is marvelous in the fact that primitive tribes attach a preferential estimate to one (or, it may be, more than one) particular number? To be sure, the nature of all the associations, individual as well as sociological, is obscure, i.e., irreducible to a logical basis. But we have at least classified the sociological phenomena with those phenomena of individual psychology that are akin to them. For that very trait

$\therefore$ This trait is shared by the present writer. 
emphasized by Lévy-Bruhl as characteristic of the sociological ideas, to wit, their initially complex character, is in the highest degree characteristic of the Galtonian phenomena. The letter $A$ is not first conceived independently by a Galtonian subject and afterward associated with a color. To the subject " $A$-brown" is an ultimate datum, "une représentation complexe," which can indeed be analyzed by the psychologist, but the analysis of which cannot, without committing the psychologist's fallacy, be projected into the subject's psychological experience.

To avoid misunderstanding, a word as to the relation of the psychological and sociological elements in a concrete case may be desirable, even at the risk of repetition. When a Crow Indian originating a new ceremony prescribes four sacred songs, his psychological condition with reference to Four may be quite different from that of an individual to whom Four appears as the incarnation of everything good and beautiful. He may be individually quite indifferent to the number Four; and even if he were not, his attitude toward it would be inextricably bound up with his attitude of unconsciously bowing to the traditional category. In other words, his psychic state is characterized, in all probability, not by a spontaneous reaction to Four, but by a spontaneous reaction to the tribal lore. Substitute Three for Four as the tribal mystic number, and his psychic reaction would not vary a jot. We may go farther. Owing to the wide distribution of Four as the mystic number, it would be rash to assume that its use as such originated with the Crow Indians. Hence we are probably dealing, not only with the sociological problems of the predetermination of individual reactions by the social group, but also with the psychological problem of a social group borrowing a cultural phenomenon from another group which, for the sake of simplicity, we will assume to be the originator. Now, within this hypothetical group, I repeat, the endowment of Four with certain attributes must somehow have taken shape in an individual mind, and the acceptance of that individual evaluation of Four-its promotion from a psychological to a cultural positionis an example of the influence of the individual on the group. That acceptance becomes the more readily intelligible when we recollect the highly hereditary character of the Galtonian phenomena and 
the fact that primitive communities are very largely constituted of blood relatives.

My general conclusion as to the relation of psychology to sociology may therefore be summarized as follows. There can be no doubt that the psychological interpretation of cultural data is fraught with serious difficulties. We have not only to disengage the psychological fact from complicating conditions of a historical order, but we must also reckon with the additional obstacle that the individual psychic phenomenon as it confronts us has already been in some way molded by sociological factors. We may, of course, cynically eschew any and every explanation of the subjective aspect of culture. If we are not content to mortify the spirit to this extent, we have no choice save between popular and scientific psychology. Scientific psychology will not solve all our sociological problems, nor many at the present time, but while not omnipotent neither is it powerless. It will not only act as a corrective in speculative interpretation, but will lend greater rigor to our formulation of fact and open new prospects of inquiry and explanation. 